\title{
The Oldest Star Clusters in the Small Magellanic Cloud
}

\author{
Kenneth J. Mighell ${ }^{1}$ \\ Kitt Peak National Observatory, National Optical Astronomy \\ Observatories, P. O. Box 26732, Tucson, AZ 85726-6732, USA \\ Ata Sarajedini ${ }^{2}$ \\ Department of Physics and Astronomy, San Francisco State University, \\ 1600 Holloway Avenue, San Francisco, CA 94132, USA
}
Rica S. French ${ }^{3}$
Middle Tennessee State University, Physics \& Astronomy Department, WPS 219, P. O. Box 71, Murfreesboro, TN 37132, USA

\begin{abstract}
We present our analysis of archival Hubble Space Telescope Wide Field Planetary Camera 2 (WFPC2) observations in F450W $(\sim B)$ and F555W $(\sim V)$ of the intermediate-age populous star clusters NGC 121, NGC 339, NGC 361, NGC 416, and Kron 3 in the Small Magellanic Cloud. We use published photometry of two other SMC populous star clusters, Lindsay 1 and Lindsay 113, to investigate the age sequence of these seven star clusters in order to improve our understanding of the formation chronology of the SMC. We analyzed the $V$ vs $B-V$ and $M_{V}$ vs $(B-V)_{o}$ color-magnitude diagrams of these populous Small Magellanic Cloud star clusters using a variety of techniques and determined their ages, metallicities, and reddenings. These new data enable us to improve the age-metallicity relation of star clusters in the Small Magellanic Cloud. In particular, we find that a closed-box continuous star-formation model does not reproduce the age-metallicity relation adequately. However, a theoretical model punctuated by bursts of star formation is in better agreement with the observational data. The full details of this analysis are reported in Mighell, Sarajedini, \& French (1998, AJ, 116, 2395).
\end{abstract}

\footnotetext{
${ }^{1}$ This research was supported by a grant from the National Aeronautics and Space Administration (NASA), Order No. S-67046-F, which was awarded by the Long-Term Space Astrophysics Program (NRA 95-OSS-16).

\section{${ }^{2}$ Hubble Fellow} ${ }^{3}$ Based on research conducted at NOAO as part of the Research Experiences for Undergraduates
program.
} 